\title{
Genomic aberrations relate early and advanced stage ovarian cancer
}

\author{
Afra Zaal • Wouter J. Peyrot • P. M. J. J. Berns • \\ Maria E. L. van der Burg • Jan H. W. Veerbeek • \\ J. Baptist Trimbos • Isabelle Cadron • Paul J. van Diest • \\ Wessel N. van Wieringen - Oscar Krijgsman • \\ Gerrit A. Meijer • Jurgen M. J. Piek • \\ Petra J. Timmers $\cdot$ Ignace Vergote $\cdot$ \\ René H. M. Verheijen • Bauke Ylstra • \\ Ronald P. Zweemer . \\ On behalf of the EORTC GCG Translational Research \\ Group
}

Accepted: 16 March 2012 / Published online: 12 May 2012

(C) The Author(s) 2012. This article is published with open access at Springerlink.com

\begin{abstract}
Background Because of the distinct clinical presentation of early and advanced stage ovarian cancer, we aim to clarify whether these disease entities are solely separated by time of diagnosis or whether they arise from distinct molecular events.
\end{abstract}

\footnotetext{
Afra Zaal and Wouter J. Peyrot contributed equally to this paper.

Electronic supplementary material The online version of this article (doi:10.1007/s13402-012-0077-5) contains supplementary material, which is available to authorized users.
}

\footnotetext{
A. Zaal $(\triangle) \cdot$ W. J. Peyrot $\cdot$ J. H. W. Veerbeek $\cdot$

R. H. M. Verheijen • R. P. Zweemer

Department of Gynaecological Oncology,

University Medical Center Utrecht,

PO Box 85500, 3508 GA Utrecht, The Netherlands

e-mail: a.zaal@umcutrecht.nl
}

P. M. J. J. Berns • M. E. L. van der Burg

EORTC Gynaecological Cancer Group

Translational Research Group chair,

Avenue Mounierlaan 83/11,

1200 Brussels, Belgium

P. M. J. J. Berns • M. E. L. van der Burg

Department of Medical Oncology, Erasmus MC;

University Medical Center Rotterdam,

PO Box 2040, 3000 CA Rotterdam, The Netherlands

\section{J. B. Trimbos}

Study Coordinator EORTC-ACTION trial, Department of Gynaecological Oncology, Leiden University Medical Center, PO Box 9600, 2300 RC Leiden, The Netherlands
Methods Sixteen early and sixteen advanced stage ovarian carcinomas, matched for histological subtype and differentiation grade, were included. Genomic aberrations were compared for each early and advanced stage ovarian cancer by array comparative genomic hybridization. To study how the aberrations correlate to the

\footnotetext{
I. Cadron $\cdot$ I. Vergote

Department of Gynecologic Oncology,

University Hospitals Leuven,

Herestraat 49,

3000 Leuven, Belgium

P. J. van Diest

Department of Pathology, University Medical Center Utrecht,

PO Box 85500, 3508 GA Utrecht, The Netherlands

W. N. van Wieringen

Department of Biostatistics and Epidemiology,

VU University Medical Center,

PO Box 7057, 1007 MB Amsterdam, The Netherlands

W. N. van Wieringen

Department of Mathematics, VU University Medical Center, PO Box 7057, 1007 MB Amsterdam, The Netherlands

O. Krijgsman · G. A. Meijer • B. Ylstra

Department of Pathology, VU University Medical Center,

PO Box 7057, 1007 MB Amsterdam, The Netherlands
} 
clinical characteristics of the tumors we clustered tumors based on the genomic aberrations.

Results The genomic aberration patterns in advanced stage cancer equalled those in early stage, but were more frequent in advanced stage $(p=0.012)$. Unsupervised clustering based on genomic aberrations yielded two clusters that significantly discriminated early from advanced stage $(p=$ 0.001 ), and that did differ significantly in survival ( $p=$ 0.002 ). These clusters however did give a more accurate prognosis than histological subtype or differentiation grade. Conclusion This study indicates that advanced stage ovarian cancer either progresses from early stage or from a common precursor lesion but that they do not arise from distinct carcinogenic molecular events. Furthermore, we show that array comparative genomic hybridization has the potential to identify clinically distinct patients.

Keywords Oligonucleotide array · Ovarian neoplasms . Chromosome aberrations $\cdot$ Neoplasm staging $\cdot$ Prognosis

\section{Introduction}

Despite improved survival over the last decades, ovarian cancer is still the most lethal gynaecological malignancy in the Western world, with a 5-year overall survival of only $25 \%$ for advanced stage $[1,2]$. In contrast, patients diagnosed at an early stage have a good 5 -year overall survival rate of $93 \%$. Early stage ovarian cancer, however, is only diagnosed in $19 \%$ of the patients.

Like other malignancies, epithelial ovarian cancer presumably results from an accumulation of genomic aberrations [3], but the exact molecular pathways by which these tumors develop have not been fully elucidated $[4,5]$. For other tumors, such as cervical and anal cancer, intraepithelial neoplasia is known to be a precursor lesion. However, no clear precursor lesion is known for ovarian cancer. Therefore, the first clinical entity to study carcinogenesis of ovarian cancer in patients is minimal localized cancer (FIGO stage I disease). As a small percentage of the patients is diagnosed with early stage ovarian cancer, it has been hypothesized that early and advanced stage ovarian cancers are two distinct subtypes. Early stage ovarian cancer (with a good prognosis) may represent a distinct biological entity with low metastatic potential and would thus arise through a different carcinogenic

\footnotetext{
J. M. J. Piek

Comprehensive Cancer Center South location TweeSteden hospital,

Dr. Deelenlaan 5,

5042AD Tilburg, The Netherlands

P. J. Timmers

Department of Gynecology and Obstetrics, Maasstad Hospital, PO Box 9100 3007, AC Rotterdam, The Netherlands

e-mail: TimmersP@Maasstadziekenhuis.nl
}

pathway. It has been suggested that these early cases are molecularly distinguishable from the advanced stage ovarian cancer with a worse prognosis [6].

Copy number change is one of the key features of genetic instability in human cancer and can be measured in formalinfixed, paraffin embedded tissue (FFPE)[7]. To test the hypothesis that early and advanced stage ovarian cancers are distinct molecular pathologic entities, we used array comparative genomic hybridization (array CGH) to detect and compare genomic aberrations in matched early and advanced stage ovarian tumors. Furthermore, we study how the aberrations correlate to the clinical characteristics of the tumors[8].

\section{Materials and methods}

\subsection{Patients and tumor tissue}

FFPE primary ovarian tumor tissue of 52 early stage ovarian cancer patients (FIGO stage I), was available from 448 participants of the EORTC 'Adjuvant ChemoTherapy in Ovarian Neoplasm' (ACTION) trial [9-11]. Of these 52 samples, 17 early stage samples with high quality DNA were matched for histological subtype and grade with FIGO stage III-IV (advanced stage) ovarian cancer samples from the Departments of Pathology of the University Medical Center Utrecht and VU University Medical Center Amsterdam, The Netherlands. FIGO stage was determined by optimal staging in all but four of the patients with advanced stage cancer. Staging was optimal $(n=7)$, modified $(n=3)$ or minimal $(n=7)$ as previously described [9]. In short, optimal staging consisted of inspection and palpation of all peritoneal surfaces; biopsies of any suspect lesions for metastases; peritoneal washing; infracolic omentectomy; (blind) biopsies of right hemidiaphragm, of right and left paracolic gutter, of pelvic sidewalls, of ovarian fossa, of bladder peritoneum, and of cul-de-sac; sampling of iliac and periaortic lymph nodes. Modified comprised of everything between optimal and minimal staging. Minimal staging was only inspection and palpation of all peritoneal surfaces and the retroperitoneal area; biopsies of any suspect lesions for metastases; peritoneal washing and infracolic omentectomy.

All samples were revised by an experienced gynaecological/oncological pathologist (PvD). The tumor percentage was defined by the pathologist per individual case; mean $77.8 \%$ (SD 7.5), median $80 \%$ range [65-95]. Samples were processed anonymously in accordance with institutional ethical guidelines, and follow up was retrieved through the EORTC ACTION trial database and the hospital information system for the early and advanced stage tumors respectively. Overall and progression free survival times were calculated from the time of randomization (within 6 weeks following staging) or the date of surgery for the early and advanced stage tumors respectively. 
The age range was $27-80$ years with a mean of 52 years for early and 62 years for advanced stage carcinomas. In six cases, an exact match for grade was unavailable. In these cases we matched with one grade difference being a grade 1 early with grade 2 advanced $(n=4)$, and a grade 2 early with grade 3 advanced $(n=2)$ (Table 1$)$.

\subsection{DNA isolation and detection of genomic aberrations by array $\mathrm{CGH}$}

Tumor tissue was dissected from freshly cut $10 \mu \mathrm{m}$ FFPE sections after confirming the tumor area by parallel haematoxylin and eosin staining. DNA was isolated as previously

Table 1 Characteristics of all 32 patients included in this study. Overall (OS) and progression free survival (PFS) are calculated from time of randomization in the early (T102-T118) and time of diagnosis in advanced stage group (T302-T318). Histological subtypes are described [7, 12] using the QIAamp DNA micro kit (Qiagen, Hilden, Germany). DNA concentration and labelling quality were measured using NanoDrop 1000 Spectrophotometer (Thermo Scientific, Wilmington, DE, USA). Labelling with cyanine 3-dUTP (Cy3) and cyanine 5-dUTP (Cy5) nucleotides was performed using the array CGH labelling kit for oligo arrays according to the manufacturer's protocol (Enzo Life Sciences, Farmingdale, NY, USA). DNA isolated from blood obtained from eighteen healthy females was pooled for use as a normal reference [13]. Free nucleotides were removed using the MinElute PCR Purification Kit (Qiagen). DNA was hybridized to $105 \mathrm{~K}$ whole genome Oxford design microarrays containing over 99.000 unique in situ synthesized 60-mer

indicated as clearcell (C), endometrioid (E), mucinous (M) or serous (S). Adjuvant Chemotherapy (CT) was administered to a subset of patients. The results of the unsupervised clustering analysis are displayed as cluster A or B

\begin{tabular}{|c|c|c|c|c|c|c|c|c|c|c|c|}
\hline Sample & Age & Stage & Type & Grade & $\mathrm{CT}$ & Staging & Status & OS (month) & Progression & PFS (months) & Cluster \\
\hline T102 & 70 & Ic capsule ruptured & $\mathrm{C}$ & 2 & no & optimal & Alive & 42 & no & 42 & $\mathrm{~A}$ \\
\hline T103 & 61 & Ic capsule ruptured & $\mathrm{C}$ & 3 & yes & optimal & Alive & 154 & no & 154 & A \\
\hline T104 & 61 & Ia & $\mathrm{C}$ & 3 & yes & optimal & Dead & 13 & yes & 10 & $\mathrm{~A}$ \\
\hline T105 & 47 & Ia & $\mathrm{C}$ & 3 & no & minimal & Alive & 136 & no & 136 & A \\
\hline T106 & 64 & Ic capsule ruptured & $\mathrm{C}$ & 3 & no & optimal & Alive & 39 & no & 39 & A \\
\hline T108 & 41 & Ic capsule ruptured & $\mathrm{E}$ & 1 & yes & minimal & Alive & 73 & no & 73 & A \\
\hline T109 & 55 & $\mathrm{Ib}$ & $\mathrm{E}$ & 1 & yes & minimal & Alive & 137 & no & 137 & A \\
\hline T110 & 64 & Ic ascites positive & $\mathrm{E}$ & 1 & no & modified & Alive & 49 & yes & 49 & A \\
\hline T111 & 46 & Ic ovarian surface & $\mathrm{E}$ & 2 & no & minimal & Alive & 146 & no & 146 & A \\
\hline T112 & 53 & Ic capsule ruptured & $\mathrm{E}$ & 2 & yes & minimal & Alive & 44 & no & 44 & A \\
\hline T113 & 42 & Ic capsule ruptured & M & 1 & no & optimal & Alive & 69 & no & 69 & A \\
\hline T114 & 35 & Ia & M & 2 & no & optimal & Alive & 152 & no & 152 & $\mathrm{~A}$ \\
\hline T115 & 27 & Ia & $\mathrm{S}$ & 1 & yes & minimal & Alive & 65 & no & 65 & A \\
\hline T116 & 62 & Ia & S & 1 & no & minimal & Alive & 59 & no & 59 & $\mathrm{~B}$ \\
\hline T117 & 50 & Ic ascites positive & S & 1 & no & modified & Dead & 61 & yes & 7 & $\mathrm{~B}$ \\
\hline T118 & 53 & Ia ovarian surface & S & 3 & no & optimal & Alive & 175 & no & 175 & A \\
\hline T302 & 47 & III & $\mathrm{C}$ & 2 & yes & optimal & Dead & 17 & yes & 17 & $\mathrm{~B}$ \\
\hline T303 & 57 & IV & $\mathrm{C}$ & 3 & yes & incomplete & Dead & 9 & yes & 8 & $\mathrm{~B}$ \\
\hline T304 & 51 & III & $\mathrm{C}$ & 3 & yes & incomplete & Dead & 14 & yes & 14 & A \\
\hline T305 & 74 & IIIb & $\mathrm{C}$ & 3 & yes & optimal & Dead & 60 & yes & 47 & $\mathrm{~B}$ \\
\hline T306 & 48 & IIIa & $\mathrm{C}$ & 3 & yes & optimal & Alive & 104 & no & 104 & $\mathrm{~B}$ \\
\hline T308 & 45 & IV & $\mathrm{E}$ & 2 & yes & optimal & Dead & 19 & yes & 9 & $\mathrm{~B}$ \\
\hline T309 & 47 & IIIc & E & 2 & yes & optimal & Dead & 41 & yes & 21 & $\mathrm{~B}$ \\
\hline T310 & 74 & IIIb & E & 2 & yes & optimal & Dead & 57 & yes & 57 & B \\
\hline T311 & 67 & IIIc & E & 3 & yes & minimal & Dead & 61 & yes & 22 & B \\
\hline T312 & 53 & IIIb & $\mathrm{E}$ & 3 & yes & optimal & Dead & 28 & yes & 28 & B \\
\hline T313 & 67 & III & M & 1 & yes & optimal & Dead & 22 & yes & 22 & A \\
\hline T314 & 76 & IV & M & 2 & yes & optimal & Dead & 15 & yes & 13 & A \\
\hline T315 & 70 & IIIc & $\mathrm{S}$ & 1 & yes & optimal & Alive & 136 & no & 136 & A \\
\hline T316 & 76 & IV & S & 1 & yes & optimal & Alive & 22 & yes & 22 & A \\
\hline T317 & 80 & IIIc & $\mathrm{S}$ & 2 & yes & incomplete & Dead & 6 & yes & 5 & B \\
\hline T318 & 61 & IIIc & $\mathrm{S}$ & 3 & yes & unknown & Dead & 5 & yes & 12 & $\mathrm{~B}$ \\
\hline
\end{tabular}


oligonucleotides (GPL8693, Agilent Technologies, Palo Alto, CA, USA), and hybridization was performed using the oligo array CGH/Chip-Chip Hybridization Kit 25 (Agilent). Each slide contained 2 arrays and one reference sample was hybridized per three tumor samples, as previously described [13]. Samples were hybridized overnight, were washed and subsequently scanned using a microarray scanner (Agilent, G2505B). Raw data of all the arrays performed are publicly available in the GEO database (accession number GSE24418).

\subsection{Data analysis}

Array CGH quality was assessed by means of the median absolute deviation (MAD) of the $\log _{2}$ ratios of a chromosome arm without a breakpoint [14]. Whereas all MAD values of the copy number changes on the q-arm of chromosome two were between 0.17 and 0.43 , the MAD value of one advanced stage sample was 0.70 . This sample and its early stage match were therefore excluded from further analyses, leaving 16 early versus 16 advanced stage cases.
Fig. 1 The frequencies of copy number gains in 16 early and 16 advanced stage ovarian cancer samples are plotted at the top of Panel A. The frequencies were tested for a difference between both stages and the false discovery rate corrected p-value is displayed. At the bottom of Panel A the analogue is shown for copy number losses. Panel B shows the array CGH profiles of all samples grouped per stage with blue indicating a loss, black a normal and yellow a gain in DNA copy number

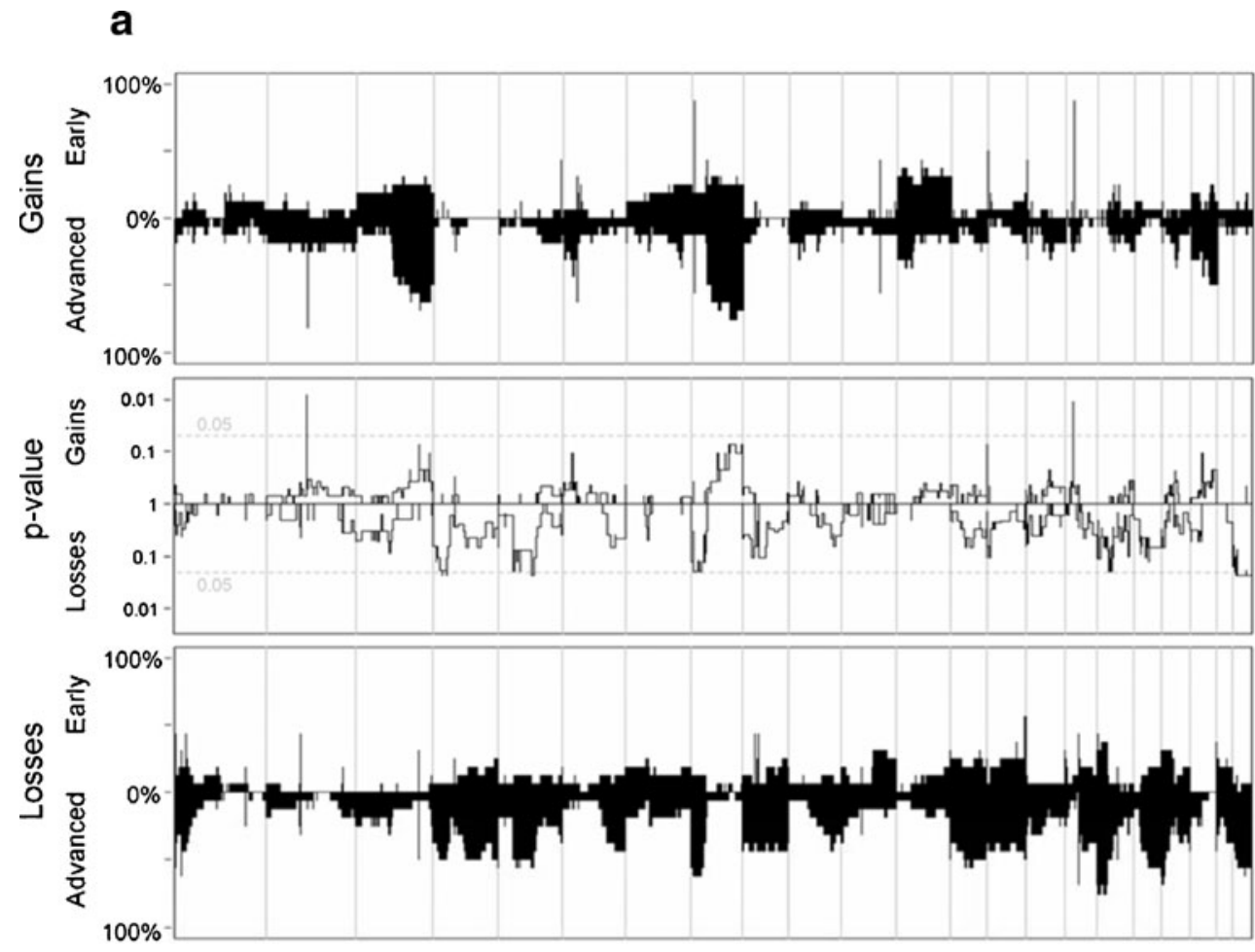

b

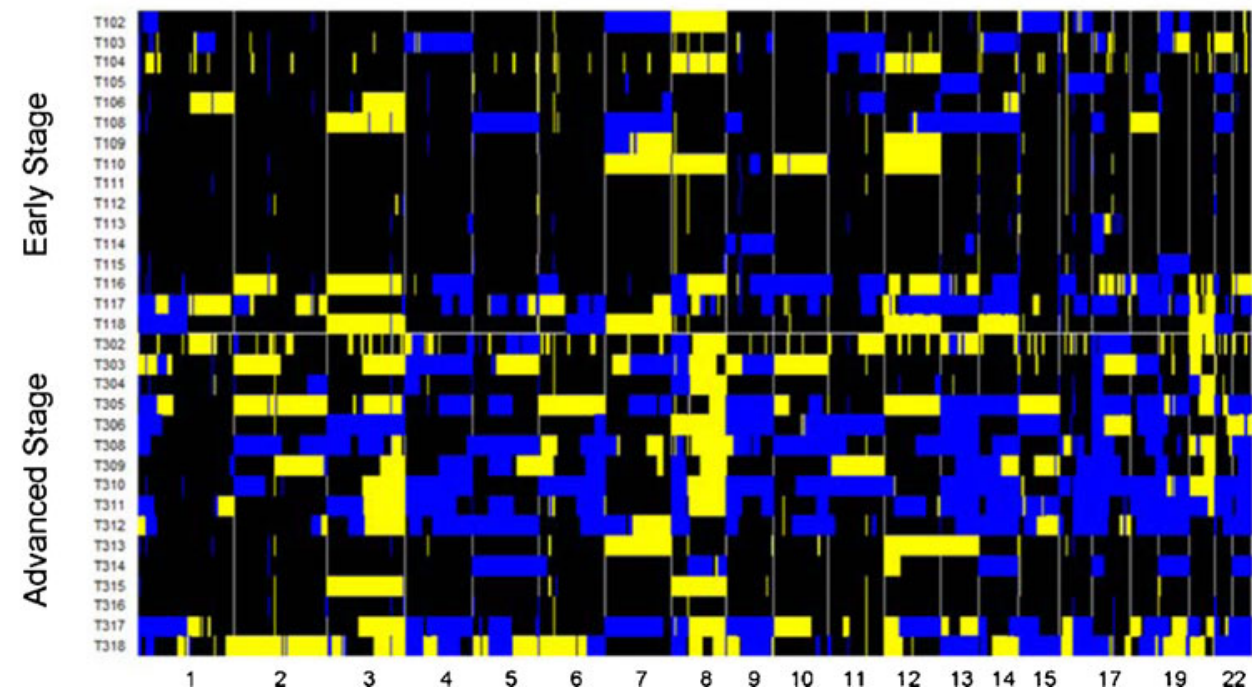


With this set of 32 samples power analysis has been performed. Log2ratios were median normalized across array [13], wave patterns were smoothed [15]. Segmentation was performed by using circular binary segmentation (CBS), since this method has shown to substantially reduce the false positives caused by the local trends in the data [16, 17]. Mode normalization was performed on the segmented data prior to automated identification of losses, gains and amplifications [16]. Dimension reduction was achieved by summarizing into regions with a threshold of 0.01 , in order to accept a maximum of $1 \%$ information loss. The frequencies of aberrations in both groups were estimated, and the false discovery rate corrected $\mathrm{p}$-values (Chi-square) of a difference in occurrence of aberrations between both groups were calculated after dividing the genome into regions [18, 19]. Using a FDR of $15 \%$, more than $50 \%$ of discriminative aberrations will be detected [20]. To test whether advanced stage progresses from early stage, we tested whether the odds for aberrations in advanced stage samples were genome-wide higher than for the early stage samples (see supplementary materials for details). Weighted unsupervised clustering of called array CGH data was performed as described previously [21, 22]. Clusters found were correlated to overall and progression free survival using KaplanMeier survival analysis with log rank testing (SPSS software package version 17.0, Chicago, IL, USA). All other analyses were performed in the statistical framework R [23].

\section{Results}

The patterns of aberrations in the advanced stage group mirrored those in the early stage group. Moreover, virtually all of the genomic aberrations were more frequent in the advanced stage group (Fig. 1a); the odds for aberrations were genome-wide significantly $(p=0.012$ higher in advanced stage ovarian cancer than in early stage disease (See supplementary materials for details). When compared on patient level (Fig. 1b) the tumor profiles are heterogeneous. However, when stratified per FIGO stage according to histological subtype (Fig. 2) the profiles display more similarity. Furthermore, Fig. 2 shows that progression of aberrations is most pronounced in the clearcell and endometrioid subtypes.

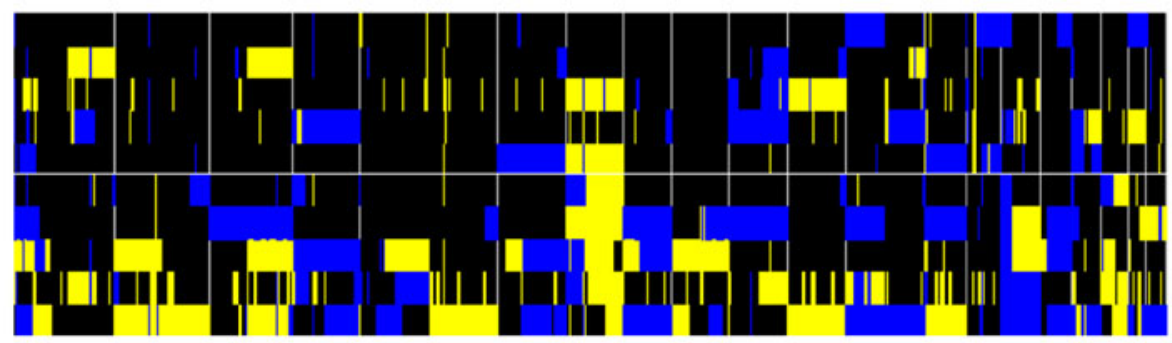

Clearcel

Early stage

Early stage

Early stage

Early stage

Early stage

Advanced stage

Advanced stage

Advanced stage

Advanced stage

Advanced stage

Endometrioid
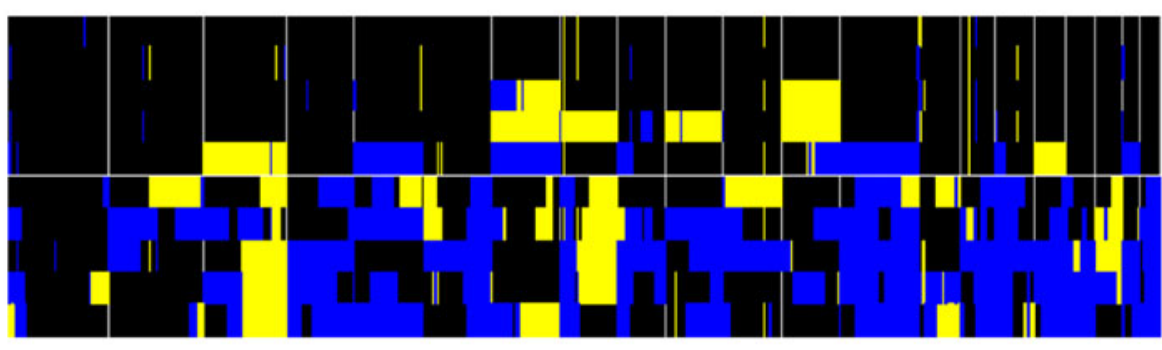

Early stage

Early stage

Early stage

Early stage

Early stage

Advanced stage

Advanced stage

Advanced stage

Advanced stage

Advanced stage

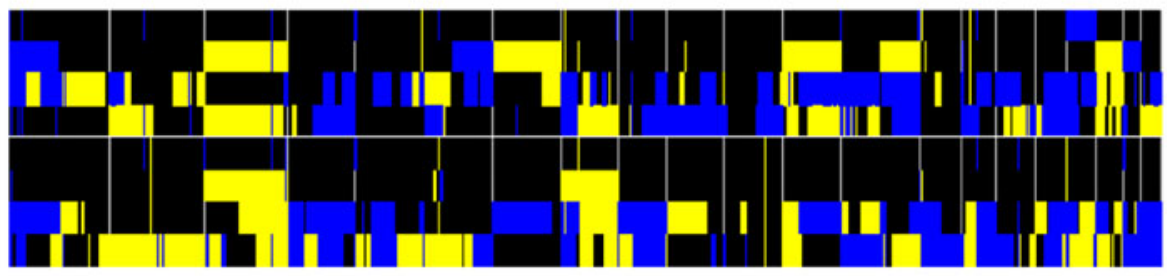

Early stage

Early stage

Early stage

Early stage

Advanced stage

Advanced stage

Advanced stage

Advanced stage

Mucinous

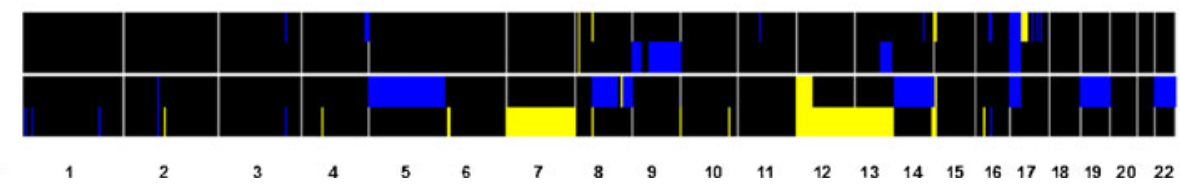

Early stage T113

Early stage T114

Advanced stage T314

Advanced stage $\quad \mathrm{T} 313$

Fig. 2 Array CGH profiles ordered per histological type and FIGO stage. Blue indicates a loss, black a normal and yellow a gain in DNA copy number 
When analysing the clinical and pathological characteristics of the tumors, we found the survival rate in patients with advanced stage ovarian cancer to be significantly lower than in patients with early stage cancer $(p<0.001)$, as expected. Contrastingly, stratification of the samples according to histological subtype yielded no significant difference in survival. However, patients with well-differentiated (grade one) tumors had significantly better survival than patients with intermediate and poorly (grades two and three) differentiated tumors combined $(p=0.044)$.

In order to study if and how aberrations correlate to the clinical behaviour of the tumors, weighted clustering of called array CGH data was performed and yielded two distinct groups of 19 tumor samples in cluster A and 13 in cluster B (Fig. 3). Cluster A contained 5 advanced and 14 early stage tumors, of which four and two, respectively had a recurrence. Cluster B contained 11 advanced and two early stage tumors of which ten and one, respectively had a recurrence (Pearson Chi-square for distribution of FIGO stage between the clusters $p=0.001)$. With a mean survival of 142.0 months (95\%CI 112.7-171.2), patients in cluster A had a significantly (Mantel Cox $\log$ rank $p=0.002$ ) better survival than patients in cluster B (43.0 months, $95 \%$ CI 27.4-58.6) (Fig. 4).

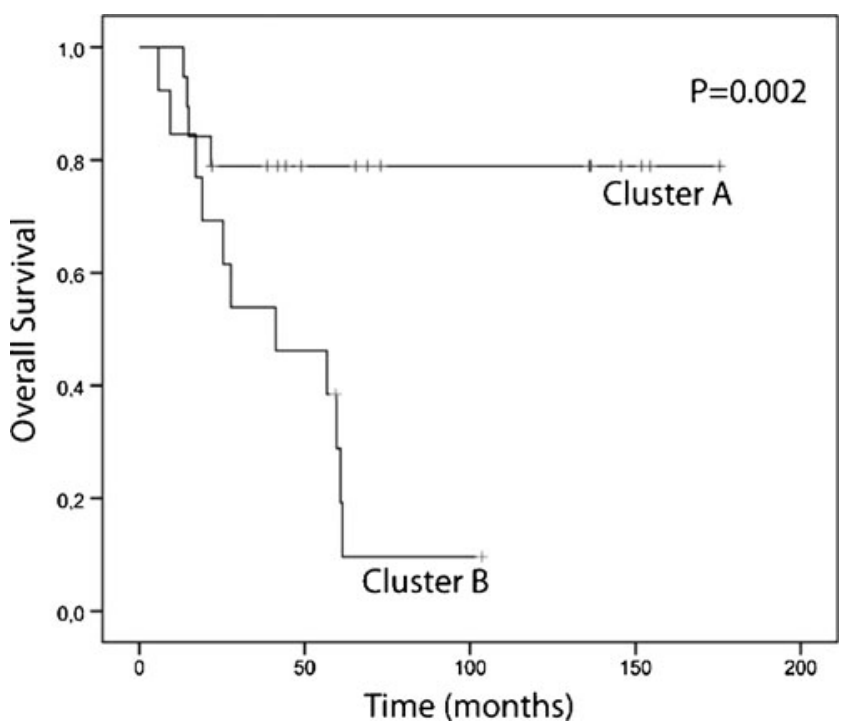

Fig. 4 Kaplan-Meier plot based on cluster A $(n=19)$ and cluster B $(n=$ 13) identified by unsupervised clustering of array CGH data. The pvalue was calculated using the Mantel-Cox log rank test

\section{Discussion}

Early and advanced stage ovarian carcinomas were compared with respect to chromosomal copy number aberrations measured by array CGH. Due to their distinct clinical

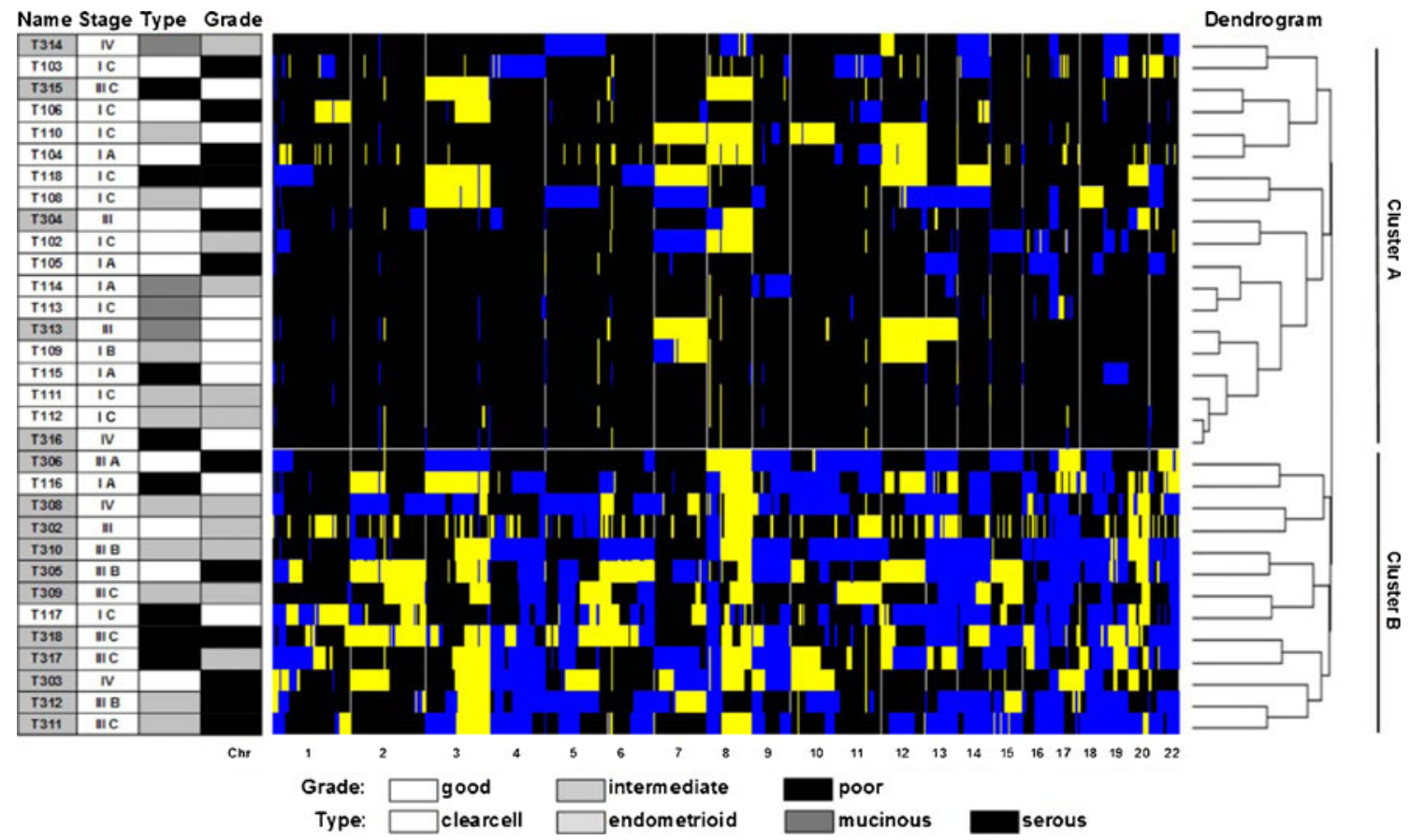

Fig. 3 Heatmap of unsupervised clustering. Blue indicates a loss, black a normal, and yellow a gain in DNA copy number. The dendrogram on the right shows the similarity between the array CGH profiles. Left of the heatmap the tumor characteristics (FIGO stage, histological type and tumor differentiation grade) are displayed. A partition of the
32 ovarian cancer patients in cluster A and cluster B is found. In cluster A samples T104, T110, T304, T313, T314 and T316 had progression. In cluster B samples T117, T302, T303, T305, T308, T309, T310, T311, T312, T317 and T318 had progression 
presentation, we hypothesised that early and advanced stage ovarian cancer develop through separate molecular pathways. Aberrations were more frequent in advanced stage and there were virtually no aberrations in early stage that were not found in advanced stage. This finding suggests that advanced stage disease either progresses from early stage disease or from a common precursor lesion. This validates earlier findings in an independent data set using low resolution array CGH [6].

Unsupervised clustering of genomic aberrations significantly discriminated early from advanced stage samples. However, to differentiate molecular subtypes based on array CGH data, independent and high-resolution validation studies are necessary.

Depending on the scoring criteria, microsatellite instability (MSI) has been reported in $0-24 \%$ of ovarian cancers [24-26], and little is known about their biological and clinical significance. However we did not test for MSI, so we can not exclude that in our samples there is MSI that could have influenced the outcome.

Ovarian carcinomas have been divided into subtypes by Shih and Kurman. [27] They propose a stratification based on clinical and histological characteristics in type I and type II tumors. Type I tumors are less frequent and are believed to be slow growing, generally confined to the ovary at diagnosis and genetically relatively stable. Histologically, type I tumors would consist of low-grade micropapillary serous carcinoma, mucinous, endometrioid, and clear cell carcinomas and would be associated with mutations in KRAS, $B R A F, P T E N$, and beta-catenin. Type II tumors would form the most common type of ovarian carcinomas and are rapidly growing, highly aggressive and genetically unstable. They would be high-grade serous carcinoma, malignant mixed mesodermal tumors and undifferentiated carcinomas and are associated with TP53 mutations. As our study is based on FIGO stage 1 tumours we cannot compare cluster A and B with type I and II. However, we show that our study population (mainly consisting of type I tumours) can be further subdivided by array CGH into patients with good and poor overall survival.

The survival times differed significantly between both clusters. This finding suggests that the comparative genomic hybridization data of an ovarian carcinoma has the potency of being of clinical value in the future.

In conclusion, in this study we showed advanced stage ovarian cancer either progresses from early stage disease or from a common precursor lesion. We reject the hypothesis that the two stages might develop through distinct carcinogenic molecular events. Furthermore, when we divided patients in two groups solely on their genomic aberrations, we found these groups to differ significantly in survival. Since copy number analysis can be performed on easy to gain pre-treatment FFPE ovarian cancer tissue, array CGH data has the potency to identify patients with a worse prognosis.

Acknowledgments This study was partly financed by the European Organisation for Research and Treatment of Cancer (EORTC, trial number 55904). We would hereby like to thank all women, investigators and their data managers who participated in the EORTC ACTION trial. Furthermore, we acknowledge Paul P. Eijk, Josien Haan, Daniëlle Israeli, Alan Mrsić, François Rustenburg and Serge J. Smeets (VU University Medical Center, Department of Pathology, Amsterdam, The Netherlands) for their technical and laboratory support.

Ethical standards This research complies with the current Dutch legislation for academic medial research.

Conflict of interest The authors declare that they have no conflict of interest.

Open Access This article is distributed under the terms of the Creative Commons Attribution License which permits any use, distribution, and reproduction in any medium, provided the original author(s) and the source are credited.

\section{References}

1. M. Akeson, A.M. Jakobsen, B.M. Zetterqvist, E. Holmberg, M. Brannstrom, G. Horvath, A population-based 5-year cohort study including all cases of epithelial ovarian cancer in western Sweden: 10-year survival and prognostic factors. Int. J. Gynecol. Cancer 19, 116-123 (2009)

2. M. Choi, C.D. Fuller, C.R. Thomas Jr., S.J. Wang, Conditional survival in ovarian cancer: results from the SEER dataset 19882001. Gynecol. Oncol. 109, 203-209 (2008)

3. B. Aunoble, R. Sanches, E. Didier, Y.J. Bignon, Major oncogenes and tumor suppressor genes involved in epithelial ovarian cancer (review). Int. J. Oncol. 16, 567-576 (2000)

4. S. Hauptmann, C. Denkert, I. Koch, S. Petersen, K. Schluns, A. Reles, M. Dietel, I. Petersen, Genetic alterations in epithelial ovarian tumors analyzed by comparative genomic hybridization. Hum. Pathol. 33, 632-641 (2002)

5. J.M. Piek, P. Kenemans, R.H. Verheijen, Intraperitoneal serous adenocarcinoma: a critical appraisal of three hypotheses on its cause. Am. J. Obstet. Gynecol. 191, 718-732 (2004)

6. V. Shridhar, J. Lee, A. Pandita, S. Iturria, R. Avula, J. Staub, M. Morrissey, E. Calhoun, A. Sen, K. Kalli, G. Keeney, P. Roche, W. Cliby, K. Lu, R. Schmandt, G.B. Mills, R.C. Bast Jr., C.D. James, F.J. Couch, L.C. Hartmann, J. Lillie, D.I. Smith, Genetic analysis of early- versus late-stage ovarian tumors. Cancer Res. 61, 5895$5904(2001)$

7. R.P. Brosens, J.C. Haan, B. Carvalho, F. Rustenburg, H. Grabsch, P. Quirke, A.F. Engel, M.A. Cuesta, N. Maughan, M. Flens, G.A. Meijer, B. Ylstra, Candidate driver genes in focal chromosomal aberrations of stage II colon cancer. J. Pathol. 221, 411-424 (2010)

8. S.J. Smeets, U. Harjes, W.N. van Wieringen, D. Sie, R.H. Brakenhoff, G.A. Meijer, B. Ylstra, To DNA or not to DNA? That is the question, when it comes to molecular subtyping for the clinic! Clin Cancer Res (2011)

9. J.B. Trimbos, I. Vergote, G. Bolis, J.B. Vermorken, C. Mangioni, C. Madronal, M. Franchi, S. Tateo, G. Zanetta, G. Scarfone, L. Giurgea, P. Timmers, C. Coens, S. Pecorelli, Impact of adjuvant 
chemotherapy and surgical staging in early-stage ovarian carcinoma: European Organisation for Research and Treatment of CancerAdjuvant ChemoTherapy in Ovarian Neoplasm trial. J. Natl Cancer Inst. 95, 113-125 (2003)

10. B. Trimbos, P. Timmers, S. Pecorelli, C. Coens, K. Ven, B.M. van der, A. Casado, Surgical staging and treatment of early ovarian cancer: Long-term analysis from a randomized trial. J Natl Cancer Inst (2010)

11. L. Verleye, P.B. Ottevanger, G.B. Kristensen, T. Ehlen, N. Johnson, M.E. van der Burg, N.S. Reed, R.H. Verheijen, K. N. Gaarenstroom, B. Mosgaard, J.M. Seoane, d. van, V, R. Lotocki, G.W. van der, B. Penninckx, C. Coens, G. Stuart, I. Vergote, Quality of pathology reports for advanced ovarian cancer: Are we missing essential information? An audit of 479 pathology reports from the EORTC-GCG 55971/NCICCTG OV13 neoadjuvant trial. Eur J Cancer (2010)

12. M.M. Weiss, M.A. Hermsen, G.A. Meijer, N.C. van Grieken, J.P. Baak, E.J. Kuipers, P.J. van Diest, Comparative genomic hybridisation. Mol. Pathol. 52, 243-251 (1999)

13. T.E. Buffart, D. Israeli, M. Tijssen, S.J. Vosse, A. Mrsic, G.A. Meijer, B. Ylstra, Across array comparative genomic hybridization: a strategy to reduce reference channel hybridizations. Genes Chromosomes Cancer 47, 994-1004 (2008)

14. T.E. Buffart, M. Tijssen, T. Krugers, B. Carvalho, S.J. Smeets, R. H. Brakenhoff, H. Grabsch, G.A. Meijer, H.B. Sadowski, B. Ylstra, DNA quality assessment for array CGH by isothermal whole genome amplification. Cell. Oncol. 29, 351-359 (2007)

15. M.A. van de Wiel, R. Brosens, P.H. Eilers, C. Kumps, G.A. Meijer, B. Menten, E. Sistermans, F. Speleman, M.E. Timmerman, B. Ylstra, Smoothing waves in array CGH tumor profiles. Bioinformatics 25, 1099-1104 (2009)

16. M.A. van de Wiel, K.I. Kim, S.J. Vosse, W.N. van Wieringen, S.M. Wilting, B. Ylstra, CGHcall: calling aberrations for array $\mathrm{CGH}$ tumor profiles. Bioinformatics 23, 892-894 (2007)

17. A.B. Olshen, E.S. Venkatraman, R. Lucito, M. Wigler, Circular binary segmentation for the analysis of array-based DNA copy number data. Biostatistics 5, 557-572 (2004)
18. M.A. van de Wiel, W.N. van Wieringen, CGHregions: dimension reduction for array $\mathrm{CGH}$ data with minimal information loss. Cancer Informat. 3, 55-63 (2007)

19. M.A. van de Wiel, S.J. Smeets, R.H. Brakenhoff, B. Ylstra, CGHMultiArray: exact P-values for multi-array comparative genomic hybridization data. Bioinformatics 21, 3193-3194 (2005)

20. I. Scheinin, J.A. Ferreira, S. Knuutila, G.A. Meijer, M.A. van de Wiel, B. Ylstra, CGHpower: exploring sample size calculations for chromosomal copy number experiments. BMC Bioinforma. 11, $331(2010)$

21. S.M. Wilting, S.J. Smeets, P.J. Snijders, W.N. van Wieringen, M. A. van de Wiel, G.A. Meijer, B. Ylstra, C.R. Leemans, C.J. Meijer, R.H. Brakenhoff, B.J. Braakhuis, R.D. Steenbergen, Genomic profiling identifies common HPV-associated chromosomal alterations in squamous cell carcinomas of cervix and head and neck1. BMC Med. Genom. 2, 32 (2009)

22. W.N. van Wieringen, M.A. van de Wiel, B. Ylstra, Weighted clustering of called array $\mathrm{CGH}$ data. Biostatistics 9, 484-500 (2008)

23. R Development Core Team, R: A language and environment for statistical computing. R Foundation for Statistical Computing, Vienna, Austria. Ref Type: Generic (2009)

24. J.P. Geisler, M.J. Goodheart, A.K. Sood, R.J. Holmes, M.A. Hatterman-Zogg, R.E. Buller, Mismatch repair gene expression defects contribute to microsatellite instability in ovarian carcinoma. Cancer 98, 2199-2206 (2003)

25. J. Helleman, I. van Staveren, W.N. Dinjens, P.F. van Kuijk, K. Ritstier, P.C. Ewing, M.E. van der Burg, G. Stoter, E.M. Berns, Mismatch repair and treatment resistance in ovarian cancer. BMC Cancer 6, 201 (2006)

26. J. Plisiecka-Halasa, A. nsonka-Mieszkowska, E. Kraszewska, A. nska-Bidzinska, J. Kupryjanczyk, Loss of heterozygosity, microsatellite instability and TP53 gene status in ovarian carcinomas. Anticancer Res. 28, 989-996 (2008)

27. I.M. Shih, R.J. Kurman, Ovarian tumorigenesis: a proposed model based on morphological and molecular genetic analysis. Am. J. Pathol. 164, 1511-1518 (2004) 